\title{
Evangelische Theologie
} ZWEIMONATSSCHRIFT

\author{
48. JAHRGANG \\ 43. JAHRGANG DER NEUEN FOLGE
}

1988

1934-1971 verantwortlich herausgegeben von Ernst Wolf

Geschäftsführender Herausgeber: Jürgen Moltmann, Tübingen Redaktion: Wolfgang Roehl, München

Herausgeber: Günter Altner, Heidelberg; Frank Crüsemann, Bethel; Helmut Gollwitzer, Berlin; Ferdinand Hahn, München; Diether Koch, Bremen; Ulrich Luz, Bern; Joachim Mehlhausen, Tübingen; Jürgen Moltmann, Tübingen; Gerhard Sauter, Bonn; Werner H. Schmidt, Bonn; Wolfgang Schrage, Bonn; Jürgen Seim, Neuwied; Theo Sundermeier, Heidelberg; Lukas Vischer, Bern

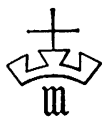

CHR. KA ISER VERLAG M Ü N C H N 


\section{THEMEN}

\section{Heft 1 Horizonte der Weltreligionen}

Michael Welker, „Einheit der Religionsgeschichte“ und „universales Selbst-

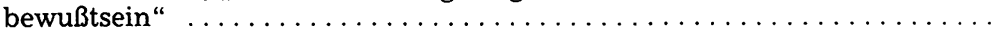
Theo Sundermeier, Gott im Buddhismus? . . . . . . . . . . . . . .

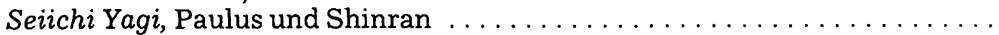
Jacques Waardenburg, Die Revitalisierung des Islam . . . . . . . . . . . . . Matthias Morgenstern, Die Heilige - von der Bedeutung Jerusalems für Juden und Moslems

\section{Heft 2 Schöpfung und Natur}

Jürgen Moltmann, Schöpfung, Bund und Herrlichkeit $\ldots \ldots \ldots \ldots \ldots$ Gunter Zimmermann, Geschöpflichkeit und Selbsterkenntnis nach Johannes Calvin

Ella Gabriel, Über zwei ontologische Kernfragen im Lichte moderner Natur-

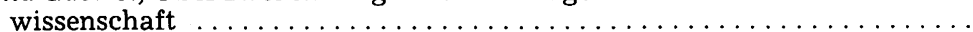

\section{Heft 3 Erfahrung und Hermeneutik}

Helmut Utzschneider, Das hermeneutische Problem der Uneindeutigkeit biblischer Texte - dargestellt an Text und Rezeption der Erzählung von Jakob am Jabbok (Gen $32,23-33) \ldots \ldots \ldots \ldots \ldots \ldots \ldots \ldots$

Albrecht Grözinger, Das „Epische“ als Aufgabe der Praktischen Theologie . Matthias J. Raden, Hermeneutik der Entsprechung oder Hermeneutik der

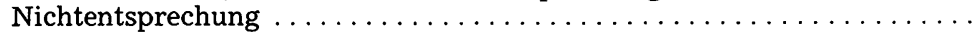

Gerhard Noller, Selbstverwirklichung oder Gottes Wirklichkeit? . . . . . .

\section{Heft 4 Altes und Neues Testament}

Christof Hardmeier, „Denn im Tod ist kein Gedenken an dich ..." (Psalm 6,6).

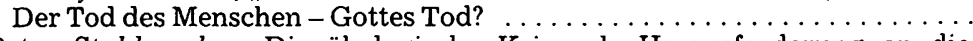

Peter Stuhlmacher, Die ökologische Krise als Herausforderung an die

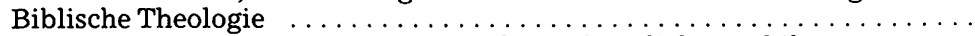
Wolfgang Schrage, Zum Komparativ in der urchristlichen Ethik . . . . . . Norbert Müller, Das Denken Martin Kählers als Beitrag zur Diskussion um die eine ",biblische Theologie“

\section{Heft 5 Kirche nach dem Holocaust}

Albert Friedlander, Zachor-Gedenke! . . . . . . . . . . . . . . Wolfgang Schrage, Meditation zu Offenbarung $2,8-11 \ldots \ldots \ldots \ldots$

Klaus Haacker, Elemente des heidnischen Antijudaismus

im Neuen Testament

Günther Baumbach, Schriftbenutzung und Schriftauswahl im Rheinischen Synodalbeschluß

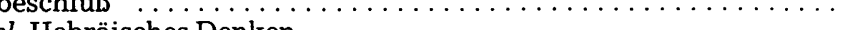

Jürgen Seim, Notizen zur Deutbarkeit des Hal $\ldots \ldots \ldots \ldots \ldots$

\section{Heft 6 Zur kirchlichen Zeitgeschichte}

Martin Greschat/Rolf Wohlrab, „Laßt Euch nicht vergiften ...“ . . . . . . . . 492 Joachim Mehlhausen, Zur Methode kirchlicher Zeitgeschichtsforschung ... 508 Martin Rohkrämer, Karl Barth

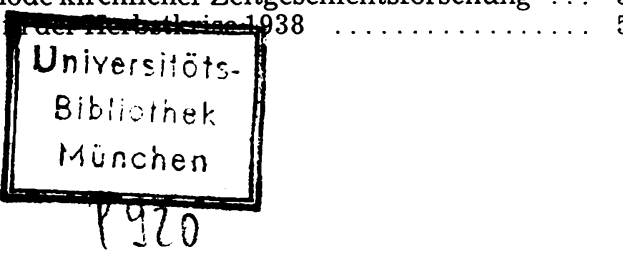


Günther Baumbach, Schriftbenutzung und Schriftauswahl im Rheinischen

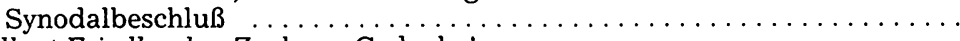

Albert Friedlander, Zachor - Gedenke! . . . . . . . . . . . . . . . . . . . . . . . .
Ella Gabriel, Über zwei ontologische Kernfragen im Lichte moderner Natur-

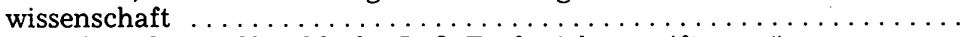

Martin Greschat/Rolf Wohlrab, „Laßt Euch nicht vergiften ..." . . . . . . .

Albrecht Grözinger, Das „Epische“ als Aufgabe der Praktischen Theologie .

Klaus Haacker, Elemente des heidnischen Antijudaismus im

Neuen Testament

Christof Hardmeier, „Denn im Tod ist kein Gedenken an dich ..." (Psalm 6,6).

Der Tod des Menschen - Gottes Tod? ......................

Joachim Mehlhausen, Zur Methode kirchlicher Zeitgeschichtsforschung . . . Otto Michel, Hebräisches Denken

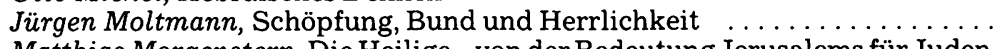

Matthias Morgenstern, Die Heilige - von der Bedeutung Jerusalems für Juden und Moslems

Norbert Müller, Das Denken Martin Kählers als Beitrag zur Diskussion um die eine „biblische Theologie" $\ldots \ldots \ldots \ldots \ldots \ldots \ldots \ldots \ldots \ldots \ldots$

Gerhard Noller, Selbstverwirklichung oder Gottes Wirklichkeit? . . . . . .

Matthias J. Raden, Hermeneutik der Entsprechung oder Hermeneutik der

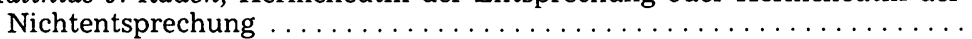

Martin Rohkrämer, Karl Barth in der Herbstkrise $1938 \ldots \ldots \ldots \ldots \ldots$

Wolfgang Schrage, Zum Komparativ in der urchristlichen Ethik . . . . . .

Wolfgang Schrage, Meditation zu Offenbarung $2,8-11 \ldots \ldots \ldots \ldots \ldots$

Jürgen Seim, Notizen zur Deutbarkeit des Holocaust . . . . . . . . . .

Peter Stuhlmacher, Die ökologische Krise als Herausforderung an die

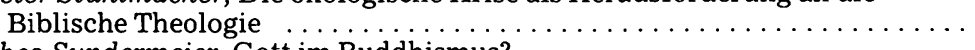

Theo Sundermeier, Gott im Buddhismus? . . . . . . . . . . . . . . .

Helmut Utzschneider, Das hermeneutische Problem der Uneindeutigkeit biblischer Texte - dargestellt an Text und Rezeption der Erzählung von

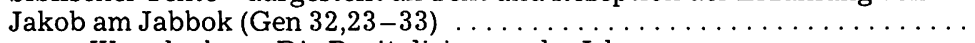

Jacques Waardenburg, Die Revitalisierung des Islam ... . . . . . . . . . . . . .

Michael Welker, „Einheit der Religionsgeschichte“ und "universales Selbst-

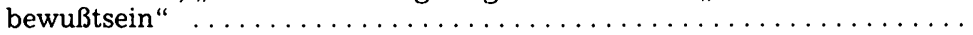

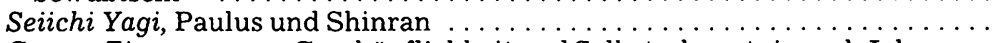

Gunter Zimmermann, Geschöpflichkeit und Selbsterkenntnis nach Johannes Calvin

\section{ZUR SITUATION}

Rainer Godel, Theorie kirchlichen Handelns $\ldots \ldots \ldots \ldots \ldots \ldots \ldots \ldots$

Anne Jensen, Theologische Forschung von Frauen $\ldots \ldots \ldots \ldots \ldots \ldots \ldots \ldots \ldots$

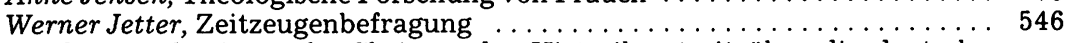

Diether Koch, Was geht Christen der Historikerstreit über die deutsche

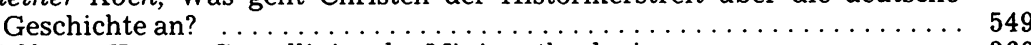

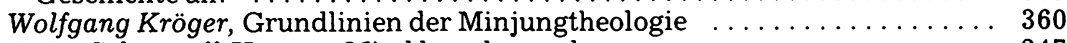

Luise Schottroff, How my Mind has changed ................. 247

Stellungnahme feministischer Theologinnen zum Vorwurf des

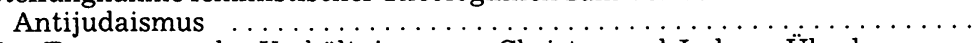

Zur Erneuerung des Verhältnisses von Christen und Juden - Überlegungen im Blick auf die 50 . Wiederkehr des Jahrestages der Synagogenbrände. (Beschluß der Landessynode der Evangelischen Kirche im Rheinland

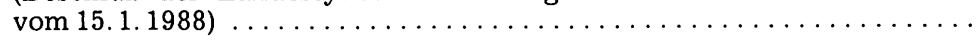




\section{KRITISCHES FORUM}

Eberhard Bethge, Christologie und Politik ............... 483

Michael von Brück, Antwort an Hans-Werner Gensichen . . . . . . . . . . 87

Hans-Werner Gensichen über Michael von Brück, Einheit der Wirklichkeit . 83

Helmut Gollwitzer, Kirchenkampf und „Judenfrage“ . . . . . . . . . 273

Siegfried Hermle, Theologiestudium in der Zeit des Nationalsozialismus . . $\quad 555$

Diether Koch, Als die Zeugen schwiegen . . . . . . . . . . . . . . . . . 480

Evi Krobath über Carter Heyward, „Und sie rührte sein Kleid an“. Eine

feministische Theologie der Beziehung $\ldots \ldots \ldots \ldots \ldots \ldots \ldots \ldots . \ldots \ldots$

Matthias Raden, Dogmatik für eine evangelische Katholizität $\ldots \ldots \ldots \ldots \quad 176$

Adelbert Schloz, Das Geheimnis der Trinität . . . . . . . . . . . . . . . . . 370

Jürgen Seim, Zwangssterilisierung und Krankenmord unter dem National-

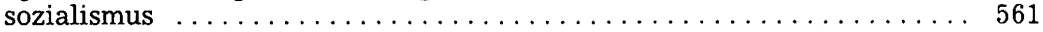

Rainer Stuhlmann, Tastende Schritte ins christologische Neuland -

Fortschritte im christlich-jüdischen Dialog? . . . . . . . . . . . . . . . . . . 474

Reinerio Arce Valentín, Christentum und Revolution: ein Gespräch mit

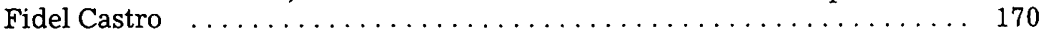

Peter Winzeler, Der Sozialismus Karl Barths in der neuesten Kritik . . . . . 262

\section{DOKUMENTATION}

Hermann Diem, Wider das Schweigen der Kirche zur Judenverfolgung . . . 471

Walther Höchstädter, Der Lemppsche Kreis . . . . . . . . . . . . . . . . . . 468

Zehn Punkte zur Selbstkontrolle christlichen Redens mit und über Juden . . 565

\section{ZU DIESEM HEFT}

Wort des geschäftsführenden Herausgebers Jürgen Moltmann . . . . . . . 1

Jürgen Moltmann, Zu diesem Heft ...................... 91

Jürgen Moltmann, Laudatio für Pastor Dr. Christian Bayers Naudé … . . . . 92

Christian Frederick Beyers Naudé, Die Krise in Südafrika als

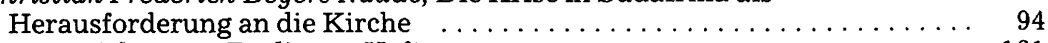

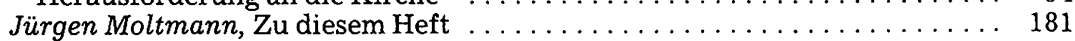

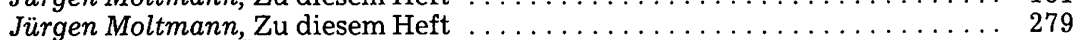

Milan Machovec, Über die Tragweite des marxistisch-christlichen Dialogs . $\quad 280$

Jürgen Seim, Zu diesem Heft $\ldots \ldots \ldots \ldots \ldots \ldots \ldots \ldots \ldots \ldots \ldots \ldots \ldots \ldots$

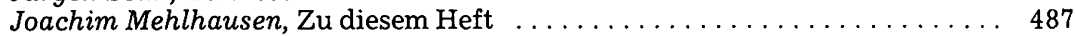

Jürgen Moltmann, Dank an Helmut Gollwitzer . . . . . . . . . . . . . . 488

Helmut Gollwitzer, „Singet dem Herrn einen neuen Gesang ... “ . . . . . . . 489 
Lieber Bruder Gensichen!

Sehr herzlich möchte ich mich bedanken für die Mühe, die Sie sich gemacht haben, „Einheit der Wirklichkeit“" zu lesen. Ich hoffe, daß Ihre einfühlende Rezension die Diskussion darüber beleben wird.

Sie werfen die grundsätzliche Frage nach "sanskritisierter" und dravidischer Kultur auf, die ja in Indien auch ein soziales Problem ist. Grundsätzlich haben Sie recht: Die brahmanische Kultur ist (nicht immer und überall) nicht die Kultur der Mehrheit. Dennoch hat sie aber, wie ich meine, Wirkungen bis in die untersten Kasten und bis hin zu den Kastenlosen gehabt, was aus kultursoziologischen Studien jüngster Zeit ex- und implizit hervorgeht und auch meinem Eindruck vor Ort entspricht (ein Beispiel: $A$. Ayrookuzhiel, The Sacred in Popular Hinduism, Bangalore [CISRS] 1983). Die Position „weder Dualismus noch Monismus" des Śankara ist, so scheint mir, ein Grundzug vieler indischer Religionstypen (keineswegs aller, wie Sie sagen), aber noch mehr: Genau dies ist die Schnittstelle des westlichen Interesses an indischer Meditation und Weltanschauung (selbstverständlich hat hier der Advaita Vedānta viel vom Buddhismus übernommen, und es ist religionsgeschichtlich nicht korrekt, denselben nur auf die Upanișaden zurückzuführen; auch Devanandan hat das noch nicht gesehen).

Ich teile nicht Schomerus' Meinung über Rāmānuja, der die holistische Gesamtschau der Trinität, die im Inkarnationsgeschehen wurzelt, m. E. nicht erreicht. Allerdings habe ich Rāmānuja nur in Übersetzungen und Sekundärquellen gelesen. Immerhin ist J. Carman der gleichen Ansicht, und ich möchte auch auf die neue Dissertation von A. W. Hunt, The Body Divine. New Perspectives in Comparative Theology with Particular Reference to Teilhard de Chardin and Rāmānuja (bei Ninian Smart in Lancaster, 1986) verweisen. Freilich interpretiere ich Śankara nicht monistisch, sondern in den Bahnen, deren Konsequenzen die bhedābheda-Schule gezogen hat. Darin folge ich Mahadevan, der hier von seinen früheren Arbeiten zu den späteren bis kurz vor seinem Tod eine Verschiebung erkennen läßt, vor allem geht dies natürlich auf meine Gespräche mit ihm zurück. Aber dies alles ist umstritten, und die gegenwärtige indische Forschung tut nicht sehr viel, um mit historisch-kritischer Analyse mehr Klarheit in die Sache zu bringen (anders die Japaner, vor allem H. Nakamura).

Natürlich kenne ich P. Devanandans ausgezeichnetes Buch über Mãyā. Warum es den Kürzungen in den Anmerkungen zum Opfer gefallen ist, weiß ich nicht. Das war ein Fehler, weil ich Devanandan viel verdanke. Inhaltlich ändert das aber nichts. Devanandan faßt m. E. zutreffend Śankaras Position auf den Seiten 206-207 zusammen und nennt drei Punkte, die Sie vermutlich im Auge haben. Er kommt zu dem gleichen Schluß wie ich, wenn er schreibt: „These three elements combined in the composite con-

Evang. Theol. 48. Jg., Heft 1, S. 87-88 ISSN 0014-3502

(c) Chr. Kaiser, 1988 
cept of Maya to give the world a pragmatic value, but no ultimate significance." (207) Und das trotz (oder wegen?) anirvacaniya. Devanandan sieht aber weniger, daß Śankkara kaum von Māyā, sondern (statistisch nachgewiesen) fast immer von avidyā spricht. Warum? Weil es nicht darum geht, eine „Ontologie“ zu entwerfen, sondern epistemologische Gesichtspunkte zu erörtern. Hier folgt er buddhistischen Argumenten, und zwar ganz in der Tradition Vasubandhus, der deshalb auch nicht einfach als „,subjektiver Idealist" bezeichnet werden kann (das trifft auf die gesamte frühe vijñānavāda-Schule zu, wie Suzuki bereits vermutet hat (Studies in the Lankkavatara-Sūtra) und Th. Kochumuttom (vom Dharmaram College) in seiner Studie „A Buddhist Doctrine of Experience“ (Motilal Banarsidass, 1982) nachweist. Wie auch immer, das Problem ist spannend und dürfte vor allem im weiteren buddhistisch- christlichen Gespräch eine Rolle spielen.

Aber das alles ist sekundär gegenüber den theologischen Fragen und Problemen. Jürgen Moltmann ist auch nicht mit meiner Leseart „perichoreuo" einverstanden, und ich gebe $z u, d a ß i c h$ mir kein letztes Urteil darüber zu erlauben wage. In der vergleichenden Sprachwissenschaft hielt man beides für möglich, und wirkungsgeschichtlich haben Sie jedenfalls recht. Inhaltlich trifft aber das "Gemeinte“, wie Sie sehr treffend sagen, „die unablässige Bewegung des einen im anderen“, doch sehr eng mit meinem Begriff der Interrelationalität zusammen? Oder irre ich mich?

Die Fragen, die Sie am Schluß stellen, erscheinen mir wie ein Katalog für die notwendige Weiterarbeit. Indem Sie Young's Buch anführen, weisen Sie schon darauf hin, daß „Einheit der Wirklichkeit“ daran leidet, daß es zu einseitig ideengeschichtlich ist und die soziale Wirkungsgeschichte von Ideen fast nicht analysiert. Das würde ich heute anders machen, aber damit ist ja schon ein wunder Punkt der klassischen Systematischen Theologie überhaupt berührt. Vielleicht könnte hier die Ökumenische Theologie bzw. die Missionswissenschaft der Systematik etwas „Entwicklungshilfe“ leisten? 